\title{
Establishment of the Effectiveness of Follow-Up Protocols for Patients under COVID-19 Epidemic
}

\author{
Juan Wang*, Meiling Zhang*, Yuanli Chen\#, Lili Li\#, Wenxiong Xu, Liang Peng \\ Deparment of Infectious Diseases, The Third Affiliated Hospital of Sun Yat-sen University, Guangzhou, China \\ Email: "cyl.ff@163.com, "22017377@qq.com,1061613246@qq.com
}

How to cite this paper: Wang, J., Zhang, M.L., Chen, Y.L., Li, L.L., Xu, W.X. and Peng, L. (2020) Establishment of the Effectiveness of Follow-Up Protocols for Patients under COVID-19 Epidemic. Open Journal of Nursing, 10, 977-988. https://doi.org/10.4236/ojn.2020.1010069

Received: September 11, 2020

Accepted: October 26, 2020

Published: October 29, 2020

Copyright $\odot 2020$ by author(s) and Scientific Research Publishing Inc. This work is licensed under the Creative Commons Attribution International License (CC BY 4.0).

http://creativecommons.org/licenses/by/4.0/

\begin{abstract}
Objectives: To control the Corona Virus Disease (COVID-19) epidemic, it is meaningful to improve the home quarantine ability and home-quarantine compliancy of patients released from medical isolation, and to prevent cross-contamination. This study aimed to up protocols for COVID-19 patients released from medical isolation. Methods: A total of 35 patients discharged from the isolation ward of the Third Affiliated Hospital of Guangzhou Sun Yat-sen University from January 22, 2020 to February 18, 2020 were included. The patients received health education through discharge preparation, education leaflets and videos. The patients were followed up on the 2nd, 7th and 14th day after discharge through WeChat and phone call. On the 2nd and 14th day after discharge, the patients completed the knowledge assessment, self-perceived burden survey and satisfaction survey using a self-made home quarantine knowledge paper. Results: There were statistical differences in the knowledge and understanding of home quarantine and self-perceived burden between the 2nd day and 14th day after home quarantine $(\mathrm{P}<0.05)$. The completion rate of home quarantine was $94.3 \%$, and the satisfaction rate was $96.7 \%$. Conclusion: By improving the discharge preparation, conducting in-time follow-up after discharge via WeChat and phone call, and performing multi-channel health education, the follow-up researchers could help the patients perform a more effective home quarantine, which could effectively prevent the spread of COVID-19 epidemic.
\end{abstract}

\section{Keywords}

Novel Coronavirus Pneumonia (COVID-19), Home Quarantine, Discharge Preparation, Follow-Up

${ }^{*}$ These authors contributed to the work equally and should be regarded as co-first authors. 


\section{Introduction}

In December 2019, multiple COVID-19 cases with a history of exposure to the South China Seafood Market were reported in Wuhan, Hubei Province, China. It was indicated that the transmission routes of this disease included contact infection, droplet and aerosol infection. It is highly infectious, with a high incidence of susceptibility crowd, and exhibits a clustering occurrence [1]. With the development of the epidemic, there are a large number of suspected or confirmed cases treating in many provinces and regions. If the body temperature is normal for three consecutive days and the virus nucleic acid testing of swab remains negative for two consecutive times (at least one day between the two tests), the suspected or confirmed cases could be released from medical isolation [2], and could go back for home quarantine. At present, home quarantine is an effective measure to control the spread of the virus. The implementation of home quarantine directly affects the health of patients and their close contacts, and also affects the control of epidemic. In order to complete the home quarantine and control the infection more effectively, the discharge preparation and follow-up of patients in the isolation ward of our hospital are done.

\section{Methods}

\subsection{Establishment of a Discharge Preparation and Follow-Up Management Team}

A discharge preparation and follow-up management team was established, and the team members included the director and the head nurse of the isolation ward, two key nurses of the infectious disease department, and a psychological consultant. Specific follow-up positions were assigned. 1) The director was responsible for the diagnosis of the patients, and the proposal of home treatment protocols; 2) the head nurse was responsible for the follow-up coordination and the management of follow-up quality; 3) the two key nurses regularly followed up the patients isolated at home after discharge, and recorded the follow-up data; 4) The psychological consultant mainly provided psychological intervention for patients with psychological problems.

\subsection{Design of Discharge Preparation and Follow-Up Protocols}

\subsubsection{Evaluation of the Patients in Isolation Wards}

Personalized nursing follow-up protocols were established based on the general conditions, education, family members, family living environment, daily living ability, and possible complications of patients. Motivational interview techniques were used [3] to understand the need of patients and their mainly caregivers after discharge, family and social support systems, and existing resources. And on this basis, home quarantine protocols were established together by the follow-up researchers and patients or their primary caregivers. The follow-up interval, content, and points were adjusted according to the different daily living abilities of patients, and a follow-up list was made (Table 1). 
Table 1. Home quarantine follow-up table of patients discharged from isolation ward.

\begin{tabular}{|c|c|}
\hline \multicolumn{2}{|c|}{ No.: __ Patient name: __ number: __ up researcher: } \\
\hline \multirow{2}{*}{ Current symptoms } & \multirow{2}{*}{$\begin{array}{l}\text { 2) Breathing difficulty 3) Cough 4) Expectoration 5) Chest distress } \\
\text { ilitation 8) Others }\end{array}$} \\
\hline & \\
\hline Sleep & Influence factors: \\
\hline Diet (appetite) & Influence factors: \\
\hline \multirow{3}{*}{ Activities and psychology } & Personal self-care condition: ADL scores \\
\hline & Exercise: 1) Yes, programs: a) square dancing b) gymnastics c) yoga d) taijiquan e) others \\
\hline & 2) No Human Communication: Self-rating happiness scale____ Self-perceived burden scale \\
\hline Hand hygiene & hand-washing method: 1) Seven-step hand-washing method 2) Washing time 3) Timing of washing hands \\
\hline Use of disinfectants & $\begin{array}{l}\text { 1) Use and storage of alcohol 2) Use of chlorine-containing disinfectants 3) Disinfection of toilet } \\
\text { 4) Floor drain treatment }\end{array}$ \\
\hline Medication compliance & 1) Regular 2) Intermittent 3) Non-drug \\
\hline Adverse drug reaction & 1) Yes__ 2) No \\
\hline $\begin{array}{l}\text { Implementation of isolation } \\
\text { measures }\end{array}$ & $\begin{array}{l}\text { 1) Use of masks 2) Single private room 3) No optional outing 4) Table-ware disinfection or private-use tableware } \\
\text { 5) A daily communication distance of }>1 \text { meter }\end{array}$ \\
\hline $\begin{array}{l}\text { Influence factors of isolation } \\
\text { measures }\end{array}$ & $\begin{array}{lll}\text { 1) Family support } & \text { 2) Career reason } & \text { 3) Others }\end{array}$ \\
\hline Return visit & \\
\hline Satisfaction & Scores (1 to 100$)$ \\
\hline \multicolumn{2}{|l|}{ Current confusion } \\
\hline Next follow-up time & Date \\
\hline
\end{tabular}

\subsubsection{Discharge Preparation}

1) Discharge preparation and predictive answers to questions

For patients who met the discharge standards [2], the physicians would patiently answer the patients' questions in the isolation ward, and explain the knowledge of COVID-19 epidemic, including the transmission routes, self-observation and judgment on the disease, measurement of body temperature and breathing exercise training [4], which deepened the patients' understanding of COVID-19 epidemic, and guide them to rationally understand this disease and make positive response to it.

2) Explanation of isolation precautions on the day of discharge

On the day of discharge, the patients were given, home quarantine notes, education leaflets, and home quarantine nursing bags which included hospital discharge education leaflets, thermometers, disposable surgical masks, latex gloves, alcohol cotton tablets and sealed bags. The patients were guided to perform real-object demonstrations, including seven-step hand washing, the use of alcohol and common chlorine-containing disinfectants (see Appendix), disinfection methods after using the toilet, use of disinfectants at home, and return visit examinations. The isolation explained to the patients: a) the isolated patients need to live in a well-ventilated single room and refuse all visits. The family members should live in different rooms, but if not, they should keep a distance of $>1 \mathrm{~m}$ away from the isolated patient. The activities of the isolated pa- 
tient should be limited. The common areas of the isolated patient and their family members should be minimized, and the common areas such as kitchens and bathrooms should be well ventilated; b) The patients' family members should prepare some essentials, such as garbage bins with lids, sealed garbage bags, multi-layer watertight paper towels for sputum cleaning, disinfection wet wipes containing chlorine or alcohol, water cups, water bottles, bedding and other daily supplies and goods for entertainment. The family members should have meals separately with their own bowls and chopsticks, which should be disinfected separately too; c) The patients should have a reasonable and balanced diet, some absorbable and fiber-rich food, such as eggs and fried vegetables, and also should eat more fruits; d) The number of caregivers should be limited. Ideally, there should be only a healthy caregiver looking after the patient. The caregivers should wear hats, masks, and disposable gloves when staying with the isolated patient. If the caregivers have touched the clothing, skin, secretions and excretions of the isolated patient, their gloves should be changed in time. When touching the things in the room of the isolated patient, the caregivers should also wash their hands using sanitizer rather than water only; e) All things such as utensils, cups and pots used by the isolated patients not be used by others, and need to be cleaned and disinfected separately in time. The virus could be effectively inactivated by hot water at $56^{\circ} \mathrm{C}$ for 30 minutes, $75 \%$ alcohol, chlorine-containing disinfectant and chloroform. Household disinfectants prepared by adding $30 \mathrm{~mL} 84$ disinfectants into $1.5 \mathrm{~L}$ water, should be used to wipe the touched goods such as door handles, bedside tables, bed frames and all non-fading furniture with duster clothes twice a day. The disinfectants should be also used to wipe or flush the bathroom and toilet surfaces at least once a day. The clothes, bed sheets, bath towels and towels of the isolated patients should be washed using ordinary laundry soap or a washing machine with ordinary laundry detergent at $60^{\circ} \mathrm{C}$ to $90^{\circ} \mathrm{C}$, and then dried. The caregivers' rooms must be ventilated at least twice a day, 30 min each time. And the ventilated time must be staggered from that of the isolated pateint's room; f) Once developing suspicious symptoms of COVID-19, such as difficulty breathing, consciousness, diarrhea and high fever, the persons must seek medical treatment in a timely manner; g) The persons with suspicious symptoms of COVID-19 should go to the fever clinic at the nearest designated hospital for medical treatment, and should wear a mask on the way to the hospital and during the medical treatment. They should not use public transportation, and should call for an ambulance or use a private vehicle. The window of the vehicle should be opened on the way to hospital. Their close contacts should keep hands clean, and should keep a distance of $>1 \mathrm{~m}$ away from other people during daily communication. When visiting a doctor, the persons should describe the illness, travel history, and residence history in details.

\subsubsection{Follow-Up}

1) Follow-up method

The patients and their family members were followed up by WeChat or phone 
call [5], and the patients strengthened their home quarantine knowledge by multi-sensory education channels such as audio, pictures and videos.

2) Follow-up time

The patients with ADL scores of $>60$, self-care ability and good family and social support were followed up on the 2nd and 14th days after discharge. For patients with ADL scores of $<60$ and good family support, follow-up could be carried out on their family members on the 2nd, 7th, and 14th day after discharge. While for patients with ADL scores of $<60$ and poor family and social support, it was necessary to contact the community, residents' committees and village committees for assistance and care, and follow-up were performed on the 2nd, 7th, and 14th day after discharge.

3) Specific follow-up content

Based on the diagnosis at hospital, the patients were mainly classified into four groups, suspected patients, mild COVID-19 patient, severe COVID-19 patients, and critically ill COVID-19 patients. Each group was managed separately, and the follow-up content of each group was different. a) After being released from medical isolation, the suspected patients were guided to perform self-observation on temperature measurement, cough, debilitation, diarrhea, sore throat, muscle pain, nasal discharge and other symptoms; b) After being discharged, the mild COVID-19 patients needed to perform routine self-observation, comply with the requirements of isolation content, and observe whether the symptoms were aggravated, as well as whether the drugs had been correctly used; c) The routine content of the severe and critically ill patients after discharge included family empowerment education for the main caregivers [6]. The empowerment education content included: the family members should be aware of care issues, and establish a care plan together with the follow-up researcher. If no family care could be provided during isolation, the follow-up researcher would contact the family members by WeChat to explain the home care plan one day before discharge, and also provide a care list. The follow-up researcher actively responsed to the issues raised by the family members, to optimize the care plan and make it concrete and feasible. The patients were guided to perform suitable movement at home according to their physical condition. With the assistance of the psychological consultant, the follow-up researcher would investigate the patients' negative emotions such as self-perceived burden, and score the self-perceived burden scale [7]. For patients with scores of $>34$ and medium-level self-perceived burden, the follow-up researcher would help them by case sharing using WeChat, and peer education. In addition, a psychiatrist would be asked to provide operable psychological treatment for the patient, such as mindfulness-based stress reduction [8], and meditation [9].

\subsection{Quality Control}

The head nurse of the isolation ward checked and supervised the work of the follow-up nurses every day, to solve the problems in time. And the nursing guidelines of discharge and follow-up guidelines of discharge preparation would 
be modified in time. Every week, the head nurse summarized the follow-up content, and forced on the confusion during follow-up, to timely control the quality, and to make improvement.

\section{Results}

\subsection{Completion of Home Quarantine}

A total of 35 patients were followed up from January 22, 2020 to February 18, 2020, including 124 Wechat follow-ups, 11 phone call follow-ups, with an average of 3.85 follow-ups. About $94.3 \%$ of patients completed the 14-days' home quarantine.

\subsection{Mastery Degree of Home Quarantine Knowledge of the Follow-up Patients}

The software SO JUMP was used to test the home quarantine knowledge of patients or the primary caregivers of patients with ADL scores of $<60$. The results on the 2 nd and 14th day after discharge were statistically described. The scores were $60.85 \pm 9.59$ on 2 nd day after discharge, and $81.12 \pm 8.8$ on the 14th day after discharge.

\subsection{The Psychological Burden of Home Quarantine Patients}

Since the patients were isolated at home, they were worried that their family members would be infected, and their financial burden increased. Therefore, the follow-up researcher also surveyed the patients' self-perceived burden through WeChat. The Self-Perceived Burden Scale (SPBS) is currently the only effective tool for measuring the self-perceived burden of patients [10]. The scale included 10 items, each scored 1 to 5 , which was classified as never (1 score), occasional (2 scores), sometimes (3 scores), mostly (4 scores), always (5 scores). The 8th item was scored reversely. The total scores of all items were calculated. The higher the score was, the heavier the patient's psychological burden was. The psychological burden level was classified as low (0 to 25 scores), medium (26 to 33 scores), and high (34 to 50 scores). An informed consent was obtained from all patients for this survey, and the survey was conducted on the 2nd and 14th day after discharge. When the patients had a total score of $>34$, a psychiatrist would be invited to provide treatment, such as meditation, and mindfulness-based stress reduction. A total of 70 survey results were collected, with a recovery rate of $100 \%$. The patient's self-perceived burden was significantly improved through follow-up and psychological guidance (Table 2).

\subsection{Survey on the Satisfaction of Home Quarantine Patients}

A survey on the follow-up satisfaction was performed on the 14th day after discharge using a self-designed questionnaire, which included 4 items, practicality of follow-up content, timeliness of confusion treatment, convenience of follow-up methods, and economy of follow-up. The total scores were 100, each 25 
Table 2. Survey on the self-perceived burden in home quarantine patients $(n=35)$.

\begin{tabular}{cc}
\hline Time (after discharge) & $\bar{X} \pm \mathrm{s}$ \\
\hline 2nd day & $41.26 \pm 5.15$ \\
14th day & $25.89 \pm 7.11$ \\
$\mathrm{t}$ & 10.84 \\
$\mathrm{p}$ & $<0.001$ \\
\hline
\end{tabular}

scores. A total of 35 surveys were recovered, with a recovery rate of $100 \%$. The patients were highly satisfied with the form and content of follow-up, and the overall evaluation was $96.7 \%$.

\section{Discussion}

\subsection{Improvement of Isolation Compliance}

They had three meals a day, and had basic supply for living, with high isolation compliance. According to Maslow's hierarchy of needs theory, only when people meet the lower level of physiological needs can they pursue and realize the higher level of needs. Among them, with good social support, higher behavioral compliance. It can be seen in this study. All patients with good family support completed the 14-days' home quarantine. During the follow-up, it was found that the main influence reason for isolation compliance was insufficient family support. Two of the patients did not complete home quarantine due to two reasons: the elderly and children were left unattended; there was no living guarantee. This reminds us that for some patients with insufficient family support, we need to cooperate with the community or welfare institutions to help care.

\subsection{Improvement of Patients' Home Quarantine Knowledge}

The comparison of the test scores between the 2nd and 14th day after discharge showed that the patients' scores were statistically significant. Multi-channel and multi-sensory education ways which transform the boring and obscure isolation knowledge into short videos and cartoons could improve the understanding and mastery of home quarantine knowledge of patients and their family members. It is more acceptable, convenient, efficient, and interactive. It is applicable to improve the compliance of people in home isolation protection. It is necessary to be carried out in a way that is easily accepted by the public for Popularization and education should, release correct, effective and practical knowledge of home isolation protection, transform knowledge into action, and play its role through media, Internet, advertising, etc.

\subsection{Reduction of Patients' Self-Perceived Burden}

The correct cognition of COVID-19 epidemic could reduce the patients' self-perceived burden. It can reduce the fear and anxiety caused by people's ignorance of things. Timely intervention of psychological consultant could en- 
hance the patients' correct understanding of negative mentality. Establish an effective mode of social psychological counseling, provide a wealth of online recreational activities to regulate the tired mood of the masses, can reduce the patients' self-perceived burden, improve the compliance of home isolation protection [11].

\subsection{Improvement of Patients' Satisfaction on Follow-Up after Discharge}

The patients could be better understood at hospital through discharge preparation. By understanding and solving the patients' problems, the researcher could establish individualized follow-up protocols for patients, which is more operable. After discharge, the patients could be followed up by WeChat, phone call, or official account, which are convenient, effective and economical. Discharge preparation and home quarantine follow-up can make it easier and more efficient for patients to acquire isolation knowledge.

\subsection{Shortcoming}

Due to the impact of the epidemic, this study only distributed questionnaires online. The number and representativeness of samples are not enough. Although in the volume design process was conducted in consultation with experts in the relevant field. Preliminary investigation, but the results are inevitably affected by the quality of the questionnaire.

In contrast to other studies. In the follow-up study, further progress should be made. Improve the survey design method, expand the sample size and improve the representativeness to increase strong applicability of the research results.

\section{Conclusion}

The patients cured and the patients released from medical isolation need be isolated at home for 14 days. Correct and effective home quarantine can help better control the spread of the virus, and prevent cross-infection. If only the treatment is emphasized without focusing on the home quarantine and follow-up after discharge, the patients would not effectively complete the home quarantine, which might result in isolation failure. The epidemic situation of COVID-19 is still serious. Except for accurate and timely medical isolation and treatment, high-quality home quarantine can help better control the epidemic. Effective discharge preparation and follow-up management are particularly important under the limited medical conditions. At the same time, we should also pay attention to the quality of patient's home quarantine, as well as their psychological changes during the period of isolation, to further improve the life quality and quarantine effect of patients at home.

\section{Grant or Support}

This study was supported by grants from Natural Science Foundation of China 
(NSFC) (No. 81570539 and 81873572).

\section{Conflicts of Interest}

The authors declare no conflicts of interest regarding the publication of this paper.

\section{References}

[1] Centers for Disease Control and Prevention (2020) 2019 Novel Coronavirus, Wuhan, China. 2019-nCoV Situation Summary. https://www.cdc.gov/coronavirus/2019-nCoV/summary.html

[2] National Health Commission Office of the People's Republic of China (2020) Diagnosis and Treatment of Novel Coronavirus Pneumonia (The 5th Trial Version, Revised Version).

[3] Rohsenow, D.J., Martin, R.A., Monti, P.M., Colby, S.M., Day, A.M., Abrams, D.B., Sirota, A.D. and Swift, R.M. (2014) Motivational Interviewing versus Brief Advice for Cigarette Smokers in Residential Alcohol Treatment. Journal of Substance Abuse Treatment, 46, 346-355. https://doi.org/10.1016/j.jsat.2013.10.002

[4] Fang, D.P., Liu, Y., Zhu, X.L., Chen, J.X., Deng, L.J., Tang, A.Z. and Zhang, W.X. (2012) Application and Effects of Six-Character Formula Respiratory Gymnastics in Continuous Nursing for Stable Patients with Chronic Obstructive Pulmonary Disease. Journal of Nursing Administration, 12, 813-815.

[5] Li, J.P. (2006) Nursing Management. 2nd Version, People's Medical Publishing House, Beijing, 141-142.

[6] Anderson, R.M. and Funnell, M.M. (2005) Patient Empowerment: Reflections on the Challenge of Fostering the Adoption of a New Paradigm. Patient Education and Counseling, 57, 153-157. https://doi.org/10.1016/j.pec.2004.05.008

[7] Arechabala, M.C., Catoni, M.I., Palma, E., et al. (2011) Depression and Self-Perceived Burden of Care by Hemodialysis Patients and Their Caregivers. Revista Panamericana de Salud Pública, 30, 74-79. https://doi.org/10.1590/S1020-49892011000700011

[8] Xiong, W.R. and Yu, L. (2011) Mindfulness Therapies: A New Kind of Psychotherapy. Medicine and Society, 24, 89-91.

[9] Wang, Y.Z. and Luo, F. (2019) Meditation Training and Its State Evaluation. Chinese Journal of Behavioral Medicine and Brain Science, 28, 177-182.

[10] Tian, F.L., Liu, H.X. and Chai, S.M. (2008) Research Progress on Self-Perceived Burden in Patients with Advanced Cancer. Chinese Journal of Nursing, 43, 955-958.

[11] Yu, H.Y., Yang, J.-Z., et al. (2013) Humanistic Care for Home Medical Observation of Close Contacts of Infectious Diseases. Journal of Nursing, 28, 82-83. 


\section{Appendix}
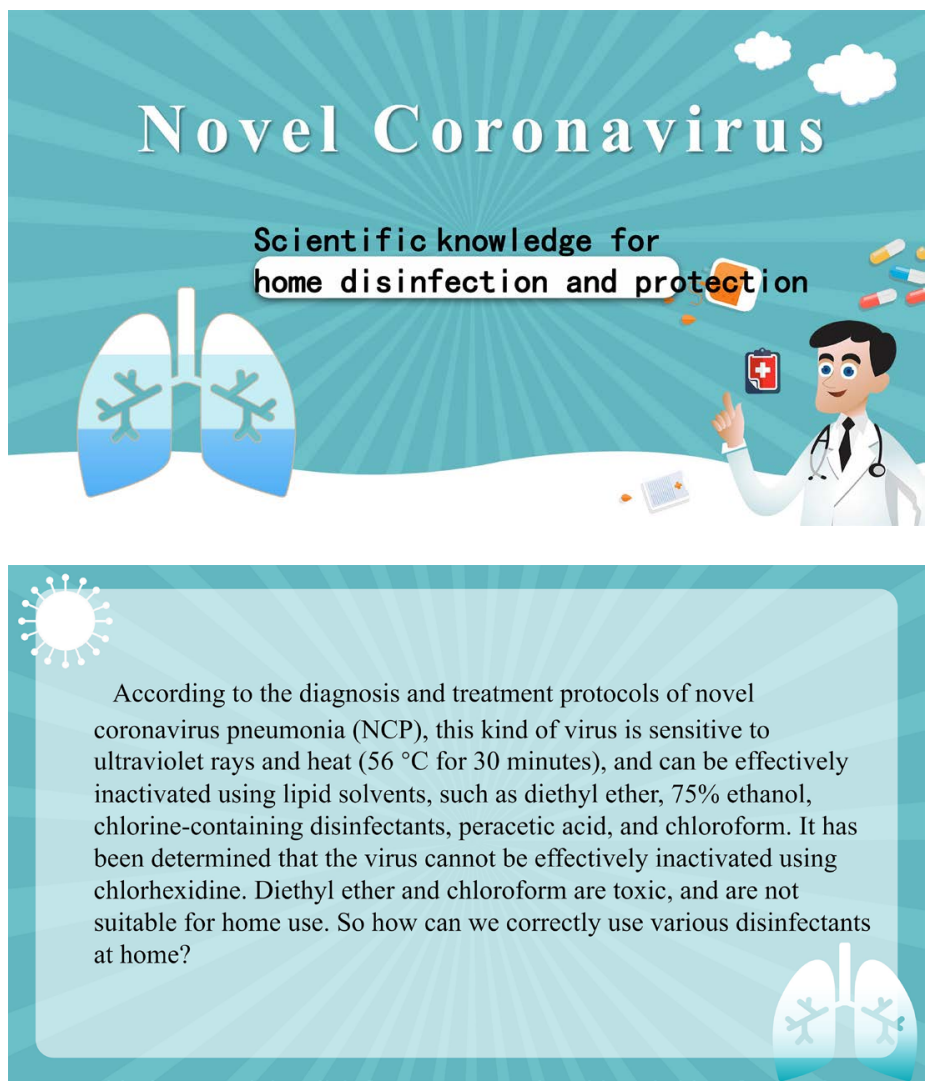

\section{Common household disinfectants 从我做起 预防新型冠状病毒}

1. Ethanol at a concentration of $75 \%$ ( $75 \%$ ethanol) Ethanol at a concentration of 70 to $75 \%$ can enter the coronavirus , thereby killing the coronavirus. The small-area high-frequency touched objects, such as door handles, mobile phones, switches, toilets, and computers, could be disinfected by wiping with $75 \%$ ethanol.
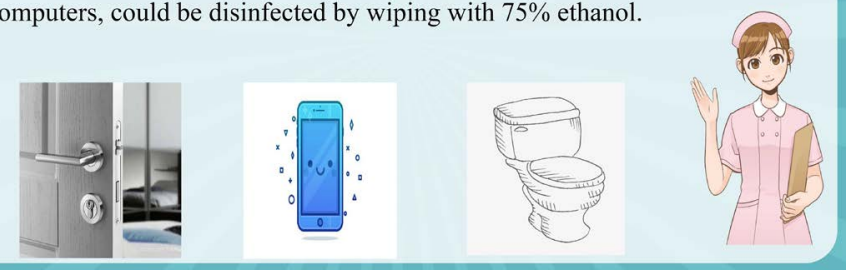

\section{* Common household disinfectants}

从我做起 预防新型冠状病毒

\section{Chlorine-containing disinfectant}

Chlorine-containing disinfectant refers to a disinfectant that can be dissolved in water to produce hypochlorous acid which could kill the microbial activity. After being diluted strictly according to the ratio instructions, chlorinecontaining disinfectants can be used to disinfect desktops, floors, plastic toys, floor drains, and feces at home. The "84" disinfectant, disinfectant powder, and chlorine-containing effervescent tablets are commonly used.
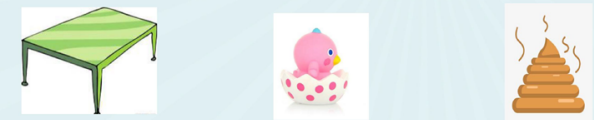


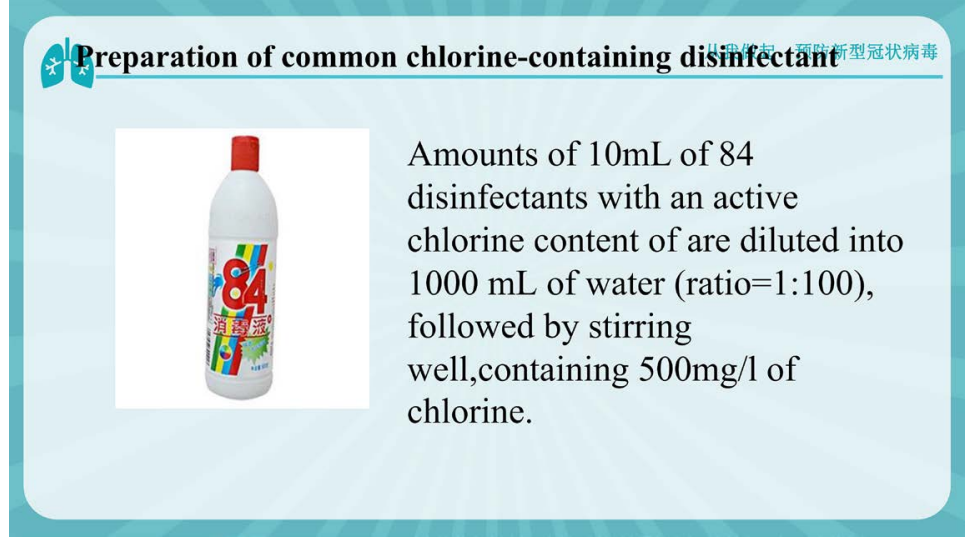

Preparation of common chlorine-containing disinfectant 状病毒 $_{\text {至 }}$

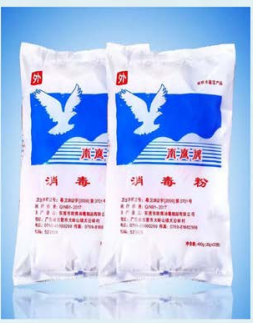

Amounts of $20 \mathrm{~g}$ of disinfection powder with an active chlorine content of 9.5 to $11.5 \%$, are well dissolved to $4000 \mathrm{~mL}$ of water (ratio=1:200).

\section{Preparation of common chlorine-containing disinfectant 状病毒 $^{-}$}
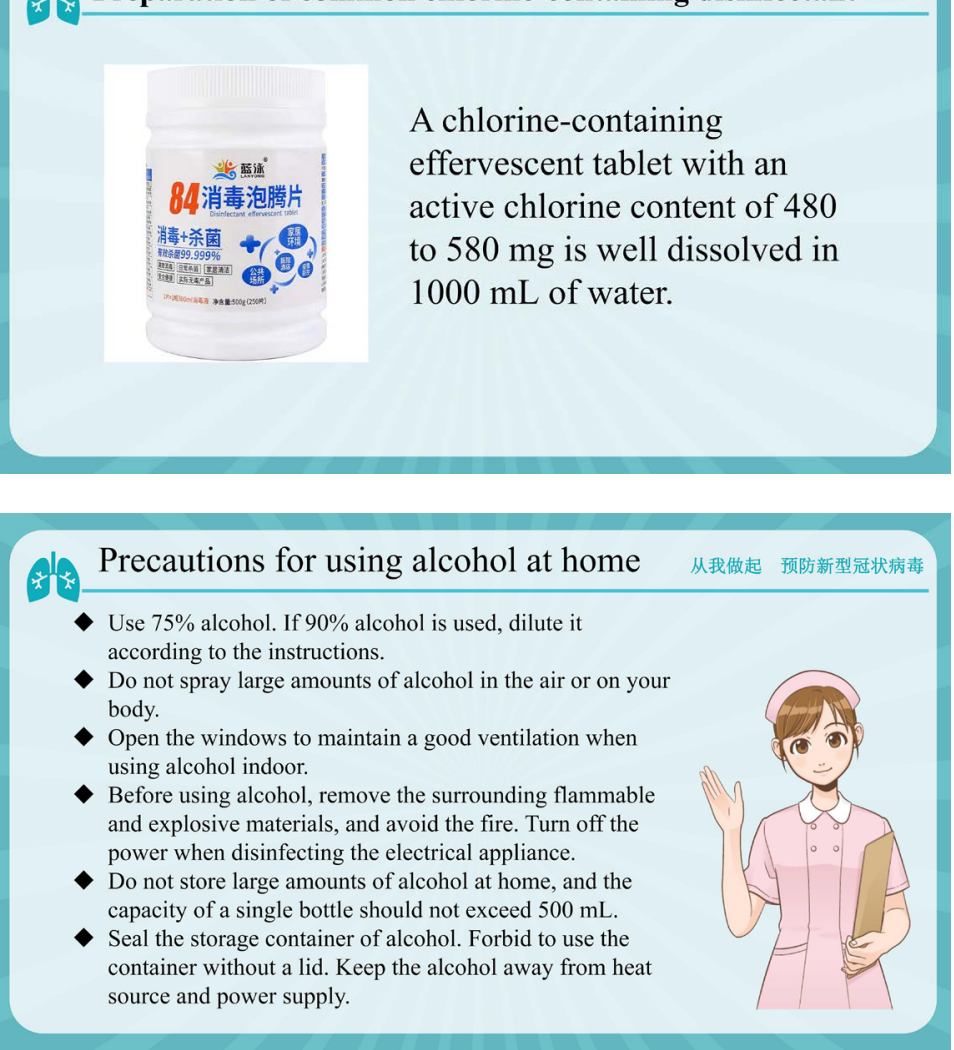
- Prepare the chlorine-containing disinfectant strictly according to the instructions.

- Avoid the skin. Wear waterproof gloves and a mask when using chlorine-containing disinfectant.

- Do not use chlorine-containing disinfectant together with toilet cleaners, since it will produce toxic gases.

- Use cold water to prepare the chlorine-containing disinfectant in a place with good ventilation, since hot water will affect the effect.

- Avoid using the chlorine-containing disinfectant and alcohol simultaneously.
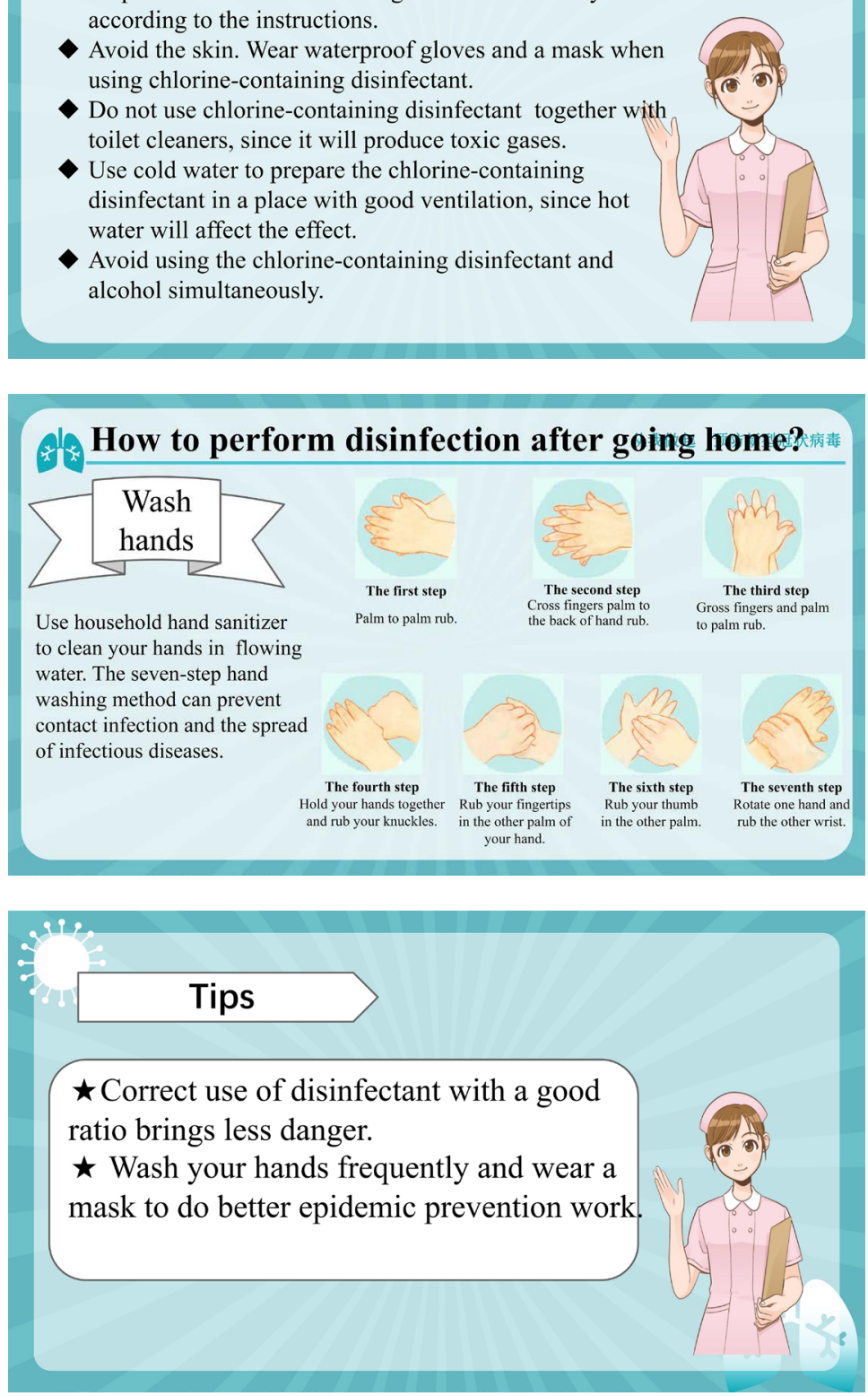